Supporting Information for

\title{
One-Pot Synthesis of Single-Crystal Palladium Nanoparticles with Controllable Sizes for Applications in Catalysis and Biomedicine
}

Yang Wang, ${ }^{\dagger \dagger}$ Alexander Biby, ${ }^{\dagger}$ Zheng Xi, ${ }^{\dagger}$ Bo Liu,,${ }^{\ddagger}$ Qinchun Rao, ${ }^{\S}$ Xiaohu Xia ${ }^{\dagger, 1, *}$

${ }^{\dagger}$ Department of Chemistry, University of Central Florida, Orlando, Florida 32816, United States

Department of Chemistry, School of Science, Beijing Jiaotong University, Beijing 100044, P. R. China

${ }^{\S}$ Department of Nutrition, Food and Exercise Sciences, Florida State University, Tallahassee, Florida 32306, United States

"NanoScience Technology Center, University of Central Florida, Orlando, Florida 32816, United States

*Corresponding author. E-mail: Xiaohu.Xia@ucf.edu 


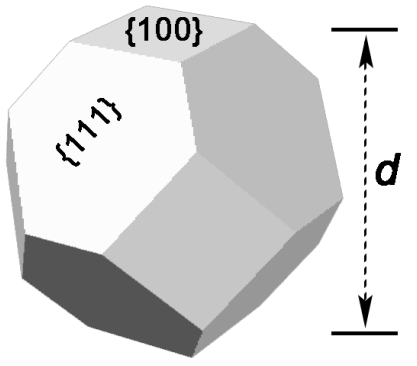

Figure S1. Schematic illustrating how the size $(d)$ of Pd nanoparticles with a truncated octahedral shape was measured in the present work. 


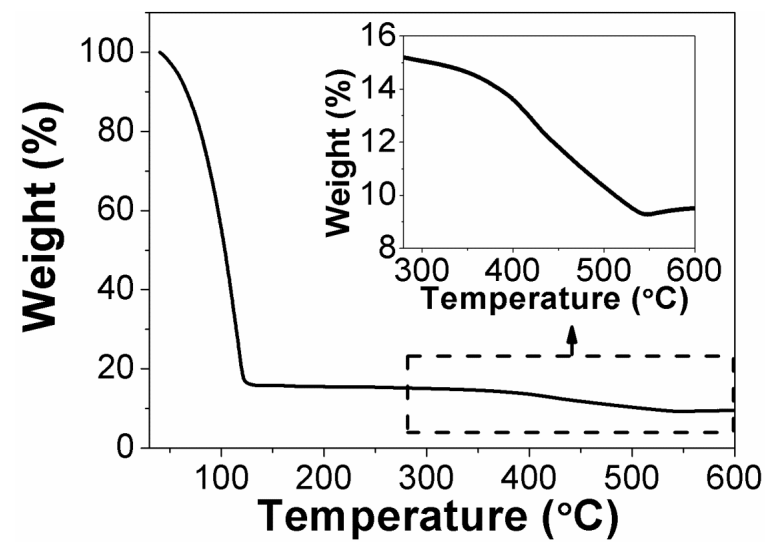

Figure S2. Normalized TGA curves for the $5.3 \mathrm{~nm}$ Pd nanoparticles shown in Figure 2. Inset is a magnified curve of the region marked with dashed box. 

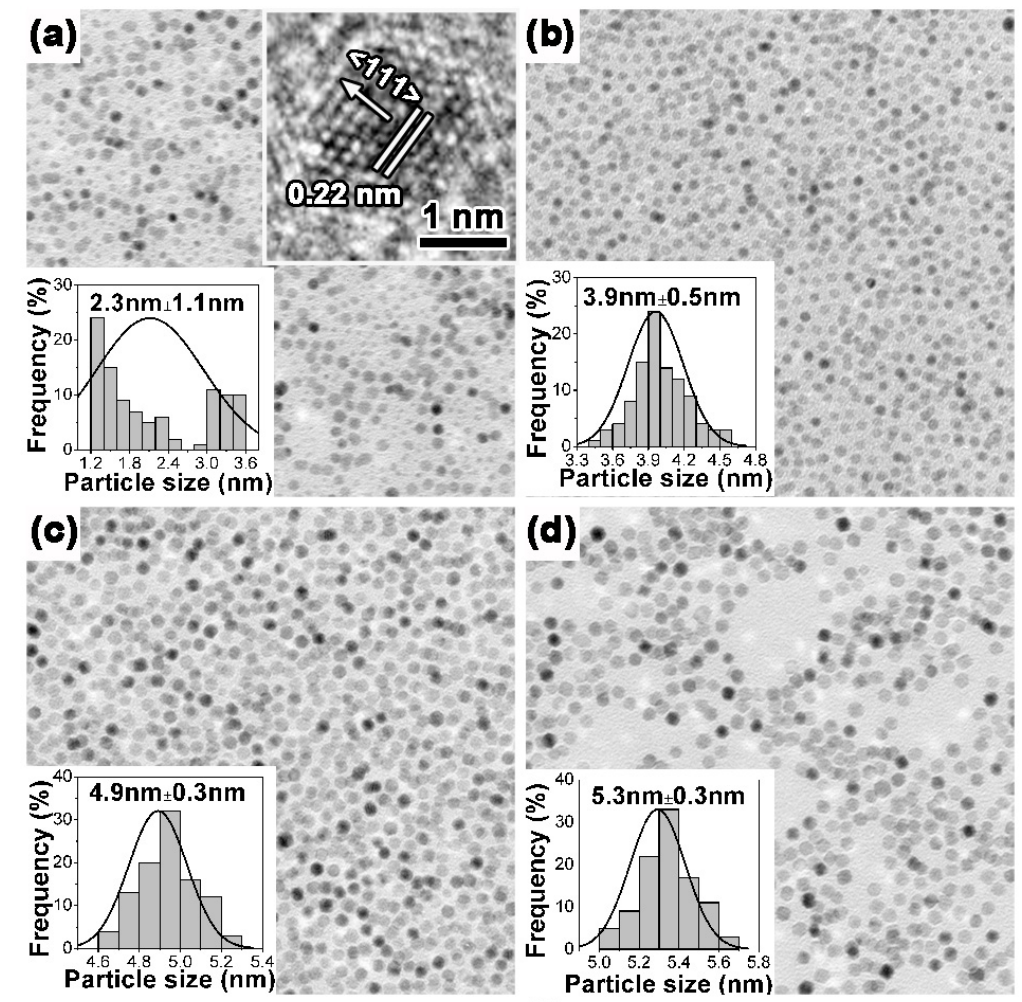

$-20 \mathrm{~nm}$

Figure S3. TEM images of four aliquots taken at different stages of a standard synthesis for the Pd nanoparticles as shown in Figure 2: (a) 10 seconds; (b) 30 seconds; (c) 5 minutes; and (d) 1 hour. Inset in (a) is the HRTEM image of a representative particle in the sample shown in (a). The curves in (a-d) show size distributions of corresponding samples. 


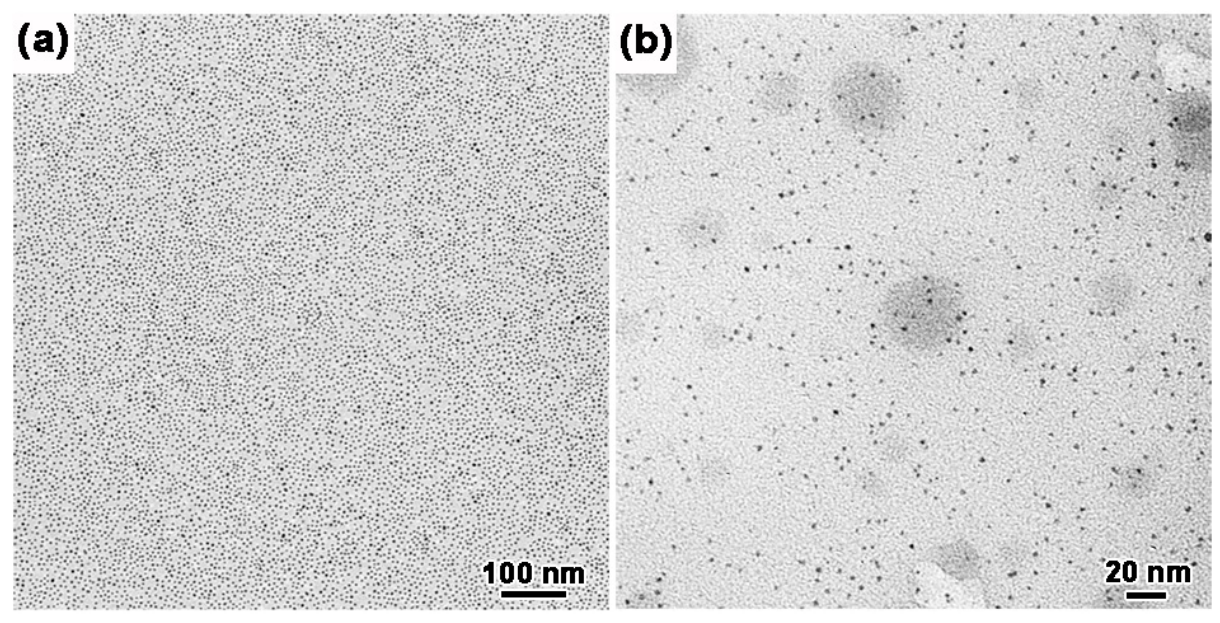

Figure S4. Low-magnification TEM images relative to the images shown in Figure 3. (a) $3.8 \mathrm{~nm}$ Pd nanoparticles; (b) $2.1 \mathrm{~nm}$ Pd nanoparticles. 

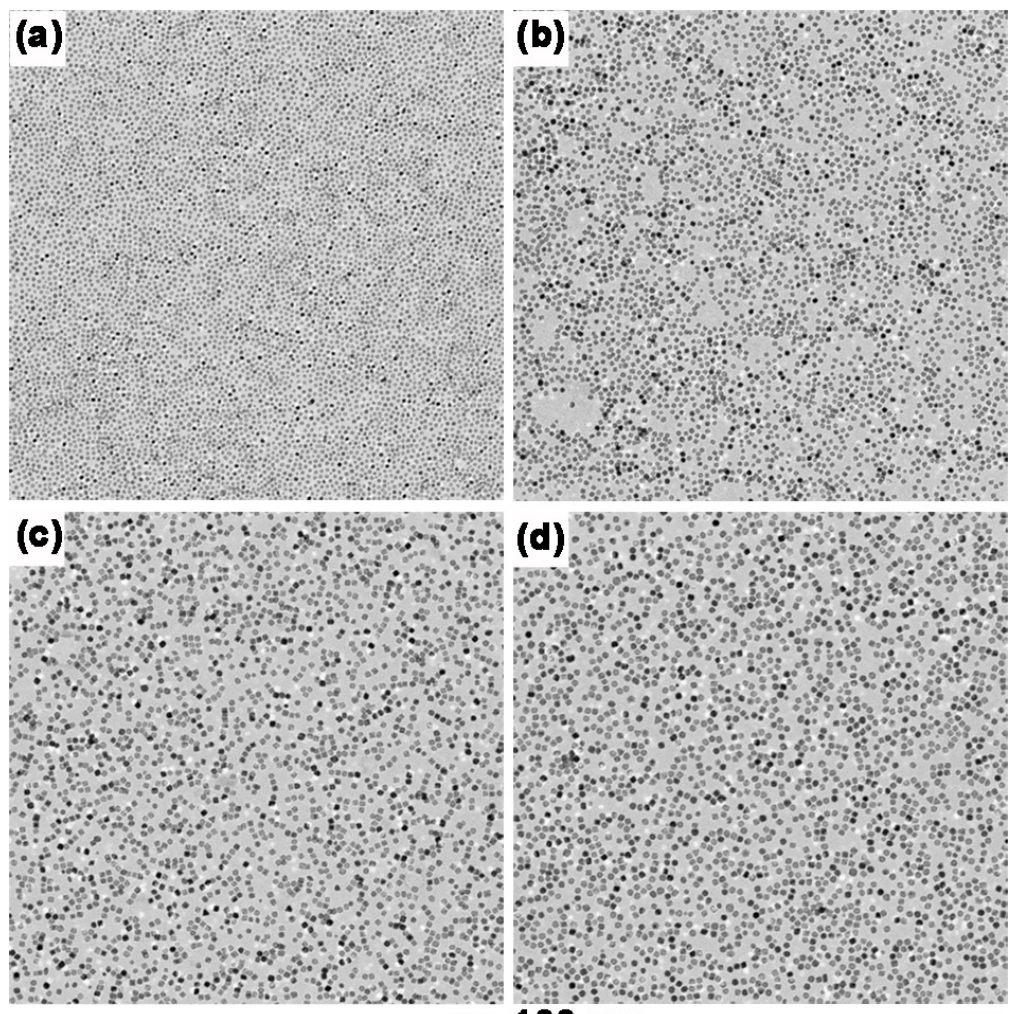

$100 \mathrm{~nm}$

Figure S5. Low-magnification TEM images relative to the images shown in Figure 4. (a) $8.0 \mathrm{~nm}$ Pd nanoparticles; (b) $10.9 \mathrm{~nm}$ Pd nanoparticles; (c) $12.8 \mathrm{~nm}$ Pd nanoparticles; (d) $14.4 \mathrm{~nm}$ Pd nanoparticles. The $100 \mathrm{~nm}$ scale bar applies to all images in (a-d). 

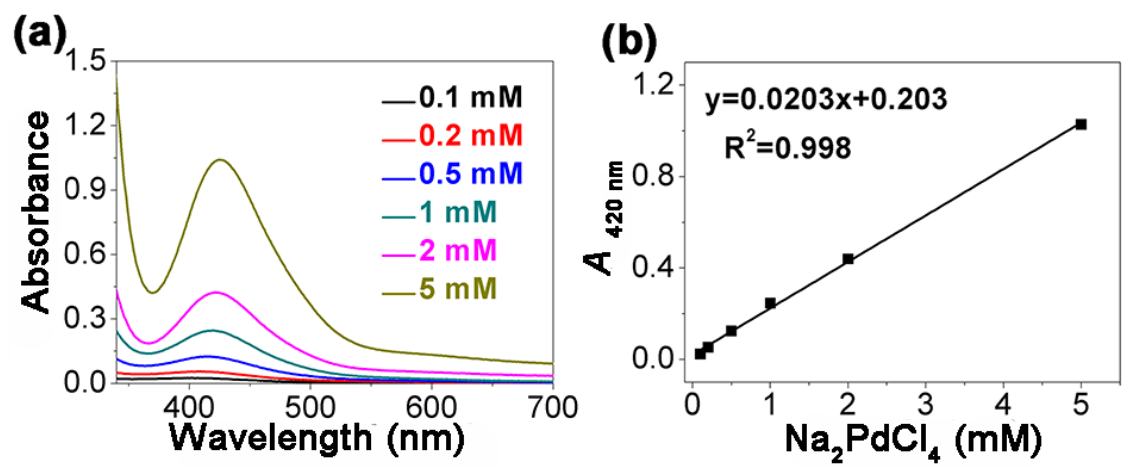

Figure S6. (a) UV-vis spectra taken from standard $\mathrm{Na}_{2} \mathrm{PdCl}_{4}$ solution of known concentrations. (b) A plot showing the absorbance at $420 \mathrm{~nm}(A 420 \mathrm{~nm})$ as a function of the concentration of $\mathrm{Na}_{2} \mathrm{PdCl}_{4}$. This calibration curve was used to quantify the concentration of $\mathrm{Na}_{2} \mathrm{PdCl}_{4}$ remaining in the reaction solution and thus to generate the plot shown in Figure 5. 


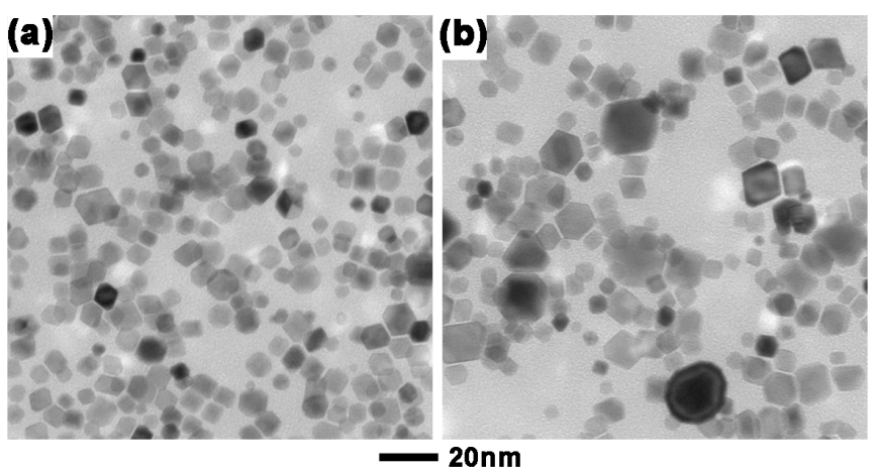

Figure S7. TEM images of Pd nanocrystals that were obtained using the standard procedure for $5.3 \mathrm{~nm}$ Pd nanoparticles, except that the amount of $\mathrm{Na}_{2} \mathrm{PdCl}_{4}$ was increased to (a) $80 \mathrm{mg}$ and (b) $160 \mathrm{mg}$. 


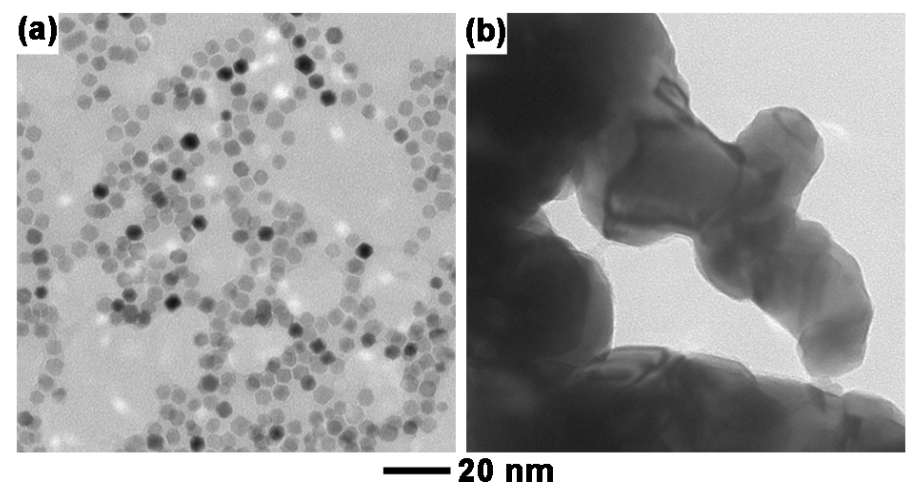

Figure S8. TEM images of Pd nanocrystals that were obtained using the standard procedure for $5.3 \mathrm{~nm}$ Pd nanoparticles, except that the amount of PVP was decreased to (a) $5 \mathrm{mg}$ and (b) $0 \mathrm{mg}$. 

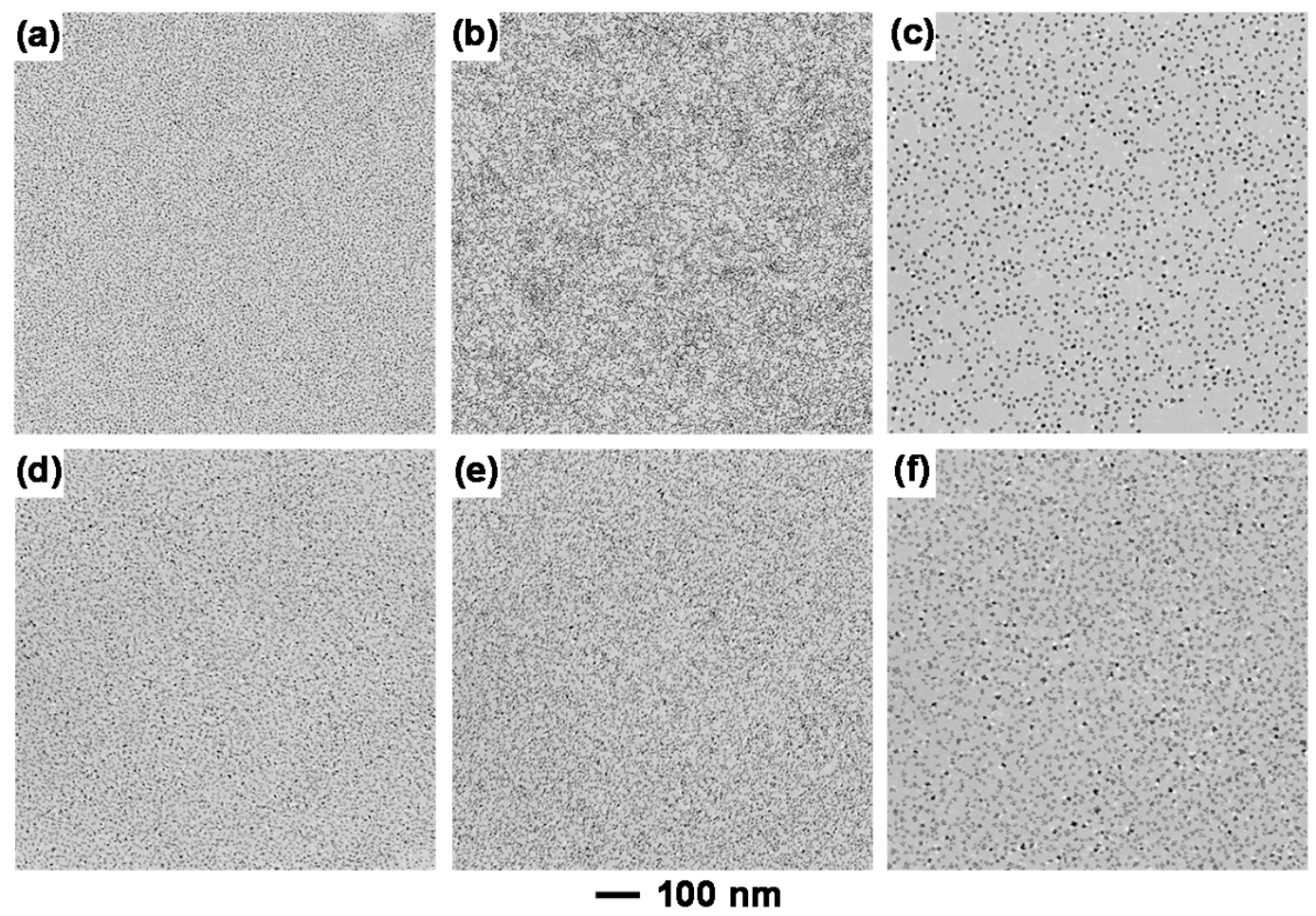

Figure S9. Low-magnification TEM images relative to the images shown in Figure 7. Each image in (a-f) corresponds to the image in Figure 7 labeled with the same letter. 